\title{
INTÉGRANDS DES NOMBRES CARACTÉRISTIQUES ET COURBURE: RIEN NE VA PLUS DĖS LA DIMENSION 6
}

\author{
JEAN PIERRE BOURGUIGNON \& ALBERT POLOMBO
}

Dédié au Professeur Buchin Su à l'occasion de son 80 ième anniversaire

\section{Introduction, motivation}

D'après la théorie de Chern-Weil (cf. [3]), les nombres caractéristiques d'une variété riemannienne compacte orientée $M$ de dimension $n=2 m$ s'expriment par des intégrales de polynôme de degré $m$ en son tenseur de courbure. Pour étudier le signe d'un nombre caractéristique, l'approche la plus simple consiste donc à regarder en chaque point le signe de l'intégrand correspondant.

En dimension 4 cette approche algébrique a permis à $J$. Thorpe puis à $N$. Hitchin d'obtenir le résultat suivant (cf. [14] et [5]) relatif aux métriques d'Einstein (i.e., celles dont la courbure de Ricci est un multiple de la métrique):

Théorème $\mathbf{E}$. Sur une variété compacte orientée d'Einstein $M$ de dimension 4 , la caractéristique d'Euler $\chi(M)$ et le premier nombre de Pontrjaguine $p_{1}(M)$ vérifient l'inégalité

$$
\left|p_{1}(M)\right| \leqslant 2 \chi(M)
$$

qui est conséquence, après intégration, de l'inégalité algébrique

$$
\left(\mathrm{AE}_{4}\right) \quad\left|p_{1}(R)\right| \leqslant 2 \chi(R),
$$

où $R$ désigne le tenseur de courbure de la métrique; de plus l'égalité n'a lieu que si la courbure de Ricci est nulle.

Il est établi dans [10] que cette inégalité est encore satisfaite si l'on impose différents types de pincement à la courbure sectionnelle ou à la courbure de Ricci, donc des conditions ouvertes sur le tenseur de courbure.

Le théorème $\mathrm{E}$ contient le théorème E'suivant (qui fut établi antérieurement par M. Berger [1]).

Received November 14, 1981. 
Théorème $\mathbf{E}_{\mathbf{4}}^{\prime} . \quad$ Si $M$ est une variété d'Einstein compacte de dimension 4, alors

$\left(\mathrm{E}_{4}^{\prime}\right)$

$$
0 \leqslant \chi(M)
$$

avec égalité si et seulement si $M$ est plate. Cette inégalité est conséquence, après intégration, de l'inégalité ponctuelle

$$
\left(\mathrm{AE}_{4}^{\prime}\right) \quad 0 \leqslant \chi(R),
$$

l'égalité ayant lieu si et seulement si $R=0$.

Cet article présente, dans une première partie, des contre-exemples à toutes les généralisations des inégalités $\left(\mathrm{AE}_{4}\right)$ et $\left(\mathrm{AE}_{4}^{\prime}\right)$ aux dimensions supérieures. Nous montrons ainsi que, si des théorèmes $\left(\mathrm{E}_{2 m}\right)$ et $\left(\mathrm{E}_{2 m}^{\prime}\right)$ existent, ils ne peuvent se démontrer par l'approche algébrique directe. En ce qui concerne l'articulation entre les théorèmes $\mathrm{E}$ et $\mathrm{E}^{\prime}$ nous montrons dans la section 1 que, pour les variétés riemanniennes de dimension $4 k$, toute généralisation du théorème $E$ implique que le théorème $E^{\prime}$ est vrai à cause de la sensibilité différente au changement d'orientation de la caractéristique d'Euler et des nombres de Pontrjaguine.

Pour justifier la deuxième partie du titre, rappelons qu'en dimension 4 le résultat suivant dû à $\mathrm{J}$. W. Milnor a aussi été obtenu par la méthode algébrique (cf. [31]).

Théorème $\mathbf{S}_{\mathbf{4}}^{\prime}$. Si $M$ est une variété riemannienne compacte de dimension 4 dont la courbure sectionnelle ne prend qu'un seul signe, alors

$$
0 \leqslant \chi(M) \text {, }
$$

inégalité qui est conséquence, après intégration, de l'inégalité ponctuelle

$$
0 \leqslant \chi(R)
$$

R. Geroch a, le premier, donné dans [4] un exemple de tenseur de courbure en dimension 6 pour lequel $\left(\mathrm{AS}_{6}^{\prime}\right)$ est violée (pour d'autres exemples, voir [2] et [7]).

Ainsi, pour les variétés riemanniennes, dès la dimension 6 ni la condition d'Einstein ni le signe de la courbure sectionnelle ne permettent d'établir, par l'approche algébrique directe, des inégalités entre nombres caractéristiques. A ce point il est intéressant de rappeler que dans [14] J. Thorpe avait donné une extension de l'inégalité $\left(\mathrm{AE}_{2 m}\right)$ dans laquelle la condition d'Einstein était remplacée par la condition d'égalité de la courbure de Lipschitz-Killing d'un $2 k$-plan et de celle de son orthogonal. Malheureusement si cette dernière condition se réduit bien à la condition d'Einstein en dimension 4 (voir [14]), elle devient extrêmement forte en dimension plus grande et n'est satisfaite par aucune classe géométriquement raisonnable de variétés riemannienries (voir 
[12] pour une discussion détaillée). De même B. Kostant avait établi que, si les valeurs propres de la courbure vue comme opérateur sur les 2-formes extérieures étaient non-négatives, alors $\left(\mathrm{AS}_{2 m}^{\prime}\right)$ était vraie (pour une preuve voir la section 1), mais cette hypothèse est, dès la dimension 4 , plus forte que celle sur le signe de la courbure sectionnelle.

Fort de ces remarques, il est naturel de considérer des classes de variétés riemanniennes plus restreintes. Nous consacrons la deuxième partie de cet article aux variétés kählériennes. Pour ces variétés les nombres de Chern s'ajoutent aux autres nombres caractéristiques. De plus la condition d'Einstein implique que $c_{1}(R)=\frac{1}{2 \pi} \rho J$ où $J$ désigne la forme de Kähler et $\rho$ la constante d'Einstein, relation qui permet de suspendre toute inégalité entre intégrands de nombres caractéristiques existant en une dimension aux dimensions supérieures. Cette remarque intervient dans le résultat suivant dû à $S$. T. Yau (cf. [16]):

Théorème. Si $M$ est une variété complexe de dimension complexe $m$ dont la première classe de Chern est négative, alors

$$
m(-1)^{m} c_{1}^{m}(M) \leqslant 2(m+1)(-1)^{m} c_{1}^{m-2}(M) c_{2}(M) .
$$

Cette inégalité renforce l'inégalité $\left(\mathrm{E}_{4}\right)$ puisque, si on oublie la structure complexe, elle signifie que $\left|p_{1}(M)\right| \leqslant \chi(M)$. A. Beauville a remarqué que pour $m=1,2$ et 3 , elle peut s'écrire

$$
\left(\mathrm{KE}_{2 m}\right) \quad \kappa^{m}(M) \leqslant(-1)^{m}(m+1)^{m} \tau(M),
$$

où $\kappa$ désigne la classes du fibré canonique et $\tau$ le genre de Todd.

Nous montrons dans la section 6 que l'inégalité $\left(\mathrm{KE}_{8}\right)$ ne peut être déduite par intégration sur $M$ de l'inégalité algébrique

$$
\left(\mathrm{AKE}_{8}\right) \quad \kappa^{4}(R) \leqslant 5^{4} \tau(R),
$$

où $R$ est un tenseur de courbure de Kähler-Einstein puisque nous donnons un contre-exemple à $\left(\mathrm{AKE}_{8}\right)$.

Nous ne discuterons pas ici de généralisation au cas kählérien du théorème $S_{4}^{\prime}$ si ce n'est pour remarquer que d'une part depuis la solution de 1 conjecture de Frankel (cf. [10] et [13]) la seule variété en jeu, lorsque la courbure est positive, est l'espace projectif $\mathbf{C P}^{m}$ et que d'autre part D. Johnson a établi le théorème $\mathrm{KS}_{6}^{\prime}$ (cf. [6]). Ce dernier résultat ne doit pas conduire à trop d'optimisme pour la généralisation à $\mathrm{KS}_{2 m}^{\prime}$ puisque, du point de vue de l'algèbre de courbure, la première dimension "stable" est la dimension complexe 4.

L'organisation de l'article est la suivante: dans la section 2 nous développons la technique de l'algèbre de courbure (cf. [8]) qui semble un outil algébrique bien adapté pour discuter des relations entre intégrands caractéristiques et 
courbure. Dans le cadre de cet article elle peut paraitre plus efficace à fournir des contre-exemples qu'à prouver des conjectures, mais elle nous semble cependant mériter un peu de publicité. Nous développons quelques remarques générales sur les inégalités entre nombres caractéristiques dans la section 3 (rôle de la caractéristique d'Euler, comportement par produit, cas de la dimension $4 k+2$ ).

Dans la section 4 nous discutons de contre-exemples riemanniens en dimensions 6 et 8, le comportement multiplicatif de la caractéristique d'Euler nous obligeant à séparer ces deux cas.

L'article se termine par la section 5 où nous traitons des variétés kählériennes. Diverses remarques utiles à des calculs généraux relatifs à de telles variétés s'y trouvent ainsi que des résultats de calculs très explicites sur un exemple particulier donnant le contre-exemple cherché.

Dans la préparation de cet article, qui s'est échelonnée sur une assez longue période, une aide précieuse nous a été apportée par le Centre de Calcul de l'Ecole Polytechnique (même si les calculs numériques ont presque disparu de cet article).

La mise au point finale a été faite lors d'un séjour à Stanford University que les auteurs remercient de son hospitalité.

\section{L'algèbre de courbure}

Soit $(V,\langle\rangle$,$) un espace vectoriel euclidien de dimension n$. L'espace vectoriel $K V=\oplus_{k} S^{2} \Lambda^{k} V$ est muni d'une loi de produit $\bullet$ définie pour $\alpha_{1} \otimes \alpha_{2}$ dans $S^{2} \Lambda^{p} V$ et $\beta_{1} \otimes \beta_{2}$ dans $S^{2} \Lambda^{q} V$ par

$$
\left(\alpha_{1} \otimes \alpha_{2}\right) \bullet\left(\beta_{1} \otimes \beta_{2}\right)=\alpha_{1} \wedge \beta_{1} \bigcirc \alpha_{2} \wedge \beta_{2}+\alpha_{1} \wedge \beta_{2} \bigcirc \alpha_{2} \wedge \beta_{1},
$$

qui en fait une algèbre, appelée algèbre de courbure (cf. [8]). Il est intéressant de noter que les espaces $\Lambda^{4 k} V$ peuvent naturellement être considérés comme sous-espaces de $S^{2} \Lambda^{2 k} V$.

Ainsi, en un point $x$ d'une variété riemannienne $(M, g)$, on peut prendre $(V,\langle\rangle)=,\left(T_{x} M, g(x)\right)$ où $T_{x} M$ est l'espace tangent en $x$. On remarque alors que la courbure de Ricci $r(x)$ appartient à $S^{2} V$ et la courbure de Riemann $R(x)$ à $S^{2} \Lambda^{2} V$. Via la métrique, $S^{2} \Lambda^{2} V$ sera considéré comme l'espace des endomorphismes symétriques de $\Lambda^{2} V$ muni de la métrique extension naturelle de celle de $V$.

Soit

$$
R=\sum_{i} \lambda_{i} \omega_{i} \otimes \omega_{i}
$$


la décomposition spectrale de l'endomorphisme $R$. La forme d'Euler de $M$ s'identifie, à un coefficient dépendant de $m$ près, à la puissance $m$-ième de $R$ dans l'algèbre de courbure, et peut donc s'écrire (cf. [8])

$$
\chi(R)=\alpha_{2 m} \sum \lambda_{i_{1}} \cdots \lambda_{i_{m}} \omega_{i_{1}} \wedge \cdots \wedge \omega_{i_{m}} \otimes \omega_{i_{1}} \wedge \cdots \wedge \omega_{i_{m}},
$$

où $\alpha_{2 m}$ est un coefficient universel dépendant de la dimension;

De même les formes de Pontrjaguine $p_{k}$ s'écrivent (cf [3], [14])

$$
p_{k}(R)=\beta_{k, 2 m} \operatorname{proj}_{\Lambda^{4 k} V}^{\perp}\left(R^{\cdot k}\right) \circ\left(R^{\cdot k}\right),
$$

où $\beta_{k, 2 m}$ est un coefficient universel dépendant de $k$ et la dimension, $\operatorname{proj}^{\perp} \cdot \Lambda^{4 k} V$ l'opérateur de projection orthogonale sur $\Lambda^{4 k} V$ vu comme sous-espace de $S^{2} \Lambda^{2 k} V$ et $\circ$ la composition des endomorphismes de $\Lambda^{2 k} V$.

On voit donc que le calcul d'une forme de Pontrjaguine d'ordre $k$ peut s'effectuer à partir des données spectrales de $R$ de la façon suivante:

(i) On calcule les produits extérieurs $k$ à $k$ des vecteurs propres de $R$. Il faut alors prendre garde que la décomposition de $R^{\cdot k}$ en somme d'endomorphismes de rang 1 obtenue à partir de ces produits n'est pas nécessairement sa décomposition spectrale, car les $2 k$-formes ainsi obtenues n'ont aucune raison de former un système libre orthonormé ne serait-ce qu'à cause de leur nombre. $C$ 'est précisément en raison de ce phénomène que les formes de Pontrjaguine rendent compte de l'irréductibilité de la métrique (et par suite les classes de Pontrjaguine de celle de la variété).

(ii) On calcule le carré de composition de $R^{\cdot k}$. Plusieurs méthodes sont possibles: soit partir de la décomposition obtenue précédemment, soit décomposer les produits obtenus sur une base orthonormée de $\Lambda^{2 k} V$. Nous préférons cette seconde méthode.

On remarquera que si

$$
R^{\bullet k}=\sum \lambda_{i_{1}} \cdots \lambda_{i_{k}} \omega_{i_{1}} \wedge \cdots \wedge \omega_{i_{k}} \otimes \omega_{i_{1}} \wedge \cdots \wedge \omega_{i_{k}}
$$

alors sa composante sur les $4 k$-formes s'écrit

$$
\operatorname{proj}_{\Lambda^{4 k} V}^{\perp}\left(R^{\cdot k}\right)=\sum \lambda_{i_{1}} \cdots \lambda_{i_{k}} \omega_{i_{1}} \wedge \cdots \wedge \omega_{i_{k}} \wedge \omega_{i_{1}} \wedge \cdots \wedge \omega_{i_{k}} .
$$

En particulier $R=\Sigma \lambda_{i} \omega_{i} \otimes \omega_{i}$ vérifie la première identité de Bianchi si et seulement si

$$
\sum \lambda_{i} \omega_{i} \wedge \omega_{i}=0
$$

On déduit ainsi de l'expression de $p_{k}$ donnée précédemment que, si $R^{\cdot k}$ admet une base de vecteurs propres décomposés, alors $p_{l}=0$ pour tout $l>k$ (comparer avec [13]). Il serait donc intéressant de trouver des conditions sur $R$ impliquant que pour un $k$ donné, les vecteurs propres de $R^{\cdot k}$ sont des 
$2 k$-formes décomposables. Cette question ne semble pas facile à résoudre à cause de la complexité des relations de Grassmann traduisant la décomposabilité dans l'algèbre extérieure.

Remarque. Dans [15] J. Vilms introduit une notion de rang pour la coubure $R$ vue comme opérateur: la courbure est dite de rang $2 r$ si $R$ applique les 2 -formes de rang 2 sur les 2 -formes de rang inférieur ou égal à $2 r$ (le rang d'une 2-forme est celui de l'endomorphisme qui lui est associé via la métrique). Cette notion ne semble pas reliée à celle du rang des vecteurs propres de $R$ : en effet $R$ peut avoir tous ses vecteurs propres de rang 2 sans que pour cela son rang au sens de $\mathbf{J}$. Vilms soit égal à 2 .

\section{Quelques remarques sur les inégalités entre nombres caractéristiques}

Nous discutons maintenant d'inégalités entre nombres caractéristiques de façon un peu générale. Supposons qu'existe une inégalité $\left(\mathrm{E}_{4 k}\right)$ généralisant $\left(\mathrm{E}_{4}\right)$ et faisant intervenir les nombres caractéristiques de la variété de façon homogène (i.e. sans terme constant). On peut séparer l'inégalité en un terme contenant les nombres de Pontrjaguine, soit $B\left(p_{1}, \cdots, p_{k}\right)(M)$, et en un terme contenant la caractéristique d'Euler, soit $a_{k} \chi(M)$, de telle sorte que l'inégalité s'écrive

$\left(\mathrm{E}_{4 k}\right)$

$$
a_{k} \chi(M)+B_{k}\left(p_{1}, \cdots, p_{k}\right)(M) \geqslant 0 .
$$

Soit $M^{\prime}$ la variété déduite de $M$ par changement d'orientation. En utilisant le comportement des nombres caractéristiques lors d'un changement d'orientation l'inégalité correspondant à $M^{\prime}$ s'écrit

$$
a_{k} \chi(M)-B_{k}\left(p_{1}, \cdots, p_{k}\right)(M) \geqslant 0 .
$$

Nous obtenons donc

$$
a_{k} \chi(M) \geqslant 0 \text {. }
$$

Nous remarquons alors que $a_{k}$ ne peut être négatif car

$$
M=S^{2} \times \cdots \times S^{2}(2 k \text { fois })
$$

est une variété d'Einstein pour laquelle $\chi(M)=2^{2 k}>0$. Si $a_{k}$ était nul, il faudrait qu'un nombre de Pontrjaguine soit nul pour toute variété de dimension $4 k$ ayant une métrique d'Einstein. Nous savons que $a_{1} \neq 0$ d'après $\left(\mathrm{E}_{4}\right)$. En dimension $4 k$ on peut effectuer le raisonnement suivant: ordonnons les classes de Pontrjaguine et faisons de même pour les monômes présents dans $B_{k}\left(p_{1}, \cdots, p_{k}\right)$ suivant l'ordre lexicographique des ordres des classes de Pontrjaguine y figurant. Ainsi $p_{3} p_{1}^{3}$ et $p_{2}^{2} p_{1}^{2}$ se représentent respectivement par 
$(3,1,1,1,0,0)$ et $(2,2,1,1,0,0)$ et par suite $p_{3} p_{1}^{3} \infty p_{2}^{2} p_{1}^{2}$. Si $\left(\theta_{1}, \cdots, \theta_{k}\right)$ avec $k \geqslant \theta_{1} \geqslant \theta_{2} \geqslant \cdots \geqslant \theta_{k} \geqslant 0$ est la suite des ordres des classes de Pontrjaguine présentes dans la monôme minimal (pour l'ordre décrit ci-dessus) de l'expression de $B_{k}\left(p_{1}, \cdots, p_{k}\right)$, alors en considérant $M=\mathbf{H} P^{\theta_{1}} \times \cdots \times \mathbf{H} P^{\theta_{k}}$, où $\mathbf{H} P^{r}$ désigne l'espace projectif quaternionien de dimension $4 r$, on obtient une contradiction. En effet sur $M$ il existe une métrique d'Einstein; par ailleurs $p_{\theta_{1}} \cdots p_{\theta_{k}}(M)$ est non nul et tout autre nombre de Pontryaguine lui est supérieur pour l'ordre $\infty$ et par suite est nul. Nous avons donc prouvé la

Proposition. S'il existe une inégalité homogène entre nombres caractéristiques vérifiée par toute variété compacte orientée de dimension $4 k$ admettant une métrique d'Einstein, alors nécessairement l'inégalité
$\left(\mathrm{E}_{4 k}^{\prime}\right)$
$0 \leqslant \chi(M)$

est vérifiée par toute variété d'Einstein compacte de dimension $4 k$.

On est ainsi conduit à se demander si pour tout tenseur de courbure d'Einstein $R$ de dimension $2 m$ on n'aurait pas
$\left(\mathrm{AE}_{2 m}^{\prime}\right)$
$0 \leqslant \chi(R)$

Nous allons montrer qu'en toute dimension paire supérieure à 4 il existe des contre-exemples à $\left(\mathrm{AE}_{2 m}^{\prime}\right)$. Le cas des dimensions non multiples de 4 est à part dans la mesure où l'intégrand d'Euler est alors un polynôme homogène de degré impair en la courbure. Par suite, si l'intégrand d'Euler satisfait une inégalité, il devrait être nul pour tout tenseur de courbure à courbure de Ricci nulle, condition évidemment très forte. Une façon de raffiner cette discussion est de prendre en compte le signe de la constante d'Einstein (i.e. le facteur de proportionnalité entre la courbure de Ricci et la métrique qui par changement homothétique de la métrique peut toujours être pris égal à $-1,0$ ou 1). Lorsque la courbure de Ricci est définie, nous pouvons normaliser en la supposant positive. Toujours à cause de l'exemple constitué par les produits de sphères, il reste à trouver des tenseurs de courbure d'Einstein à constante positive et intégrand d'Euler négatif, le cas Ricci plat étant traité à part.

Pour produire des contre-exemples dans une dimension donnée, on pense de suite à prendre des produits de variétés de dimension plus basse, la caractéristique d'Euler se comportant multiplicativement $(\chi(M \times N)=\chi(M) \chi(N))$. Par contre on n'est sûr de l'existence de métriques d'Einstein sur le produit de deux variétés d'Einstein que si les constantes d'Einstein des deux facteurs ont le même signe, restriction qui est compatible avec la normalisation faite précédemment. 
Si tout exemple de tenseur de courbure d'Einstein à constante d'Einstein positive et intégrand d'Euler négatif fournit des exemples analogues en dimension plus grande par produit avec des sphères $S^{2}$ de rayon convenablement ajusté, il n'en est pas de même d'un exemple de tenseur de courbure Ricci-plat en dimension 6 à intégrand d'Euler négatif qui ne peut se suspendre en dimension 8. En effet il faudrait faire un produit avec un facteur plat mais alors $\chi$ devient nul. Par contre dès la dimension 10 on peut effectuer un produit avec un tenseur de courbure à courbure de Ricci nulle, intégrand d'Euler positif et dimension 4. De tel tenseurs existent forcément puisque les surfaces $K 3$ ont des métriques à courbure de Ricci nulle (cf. [16]) et que leur caractéristique d'Euler vaut 24.

\section{Contre-exemples riemanniens en dimensions 6 et 8}

Nous fournissons dans cette section des contre-exemples aux inégalités $\left(\mathrm{AE}_{6}^{\prime}\right)$ et $\left(\mathrm{AE}_{8}^{\prime}\right)$ ainsi qu'à leur cas d'égalité. Pour donner de tels contre-exemples, plusieurs approches sont possibles parmi lesquelles:

(1) partir de tenseurs de courbure suggérés par la géométrie;

(2) effectuer les calculs à partir d'objets algébriques choisis pour leur simplicité.

En utilisant l'approche (1), J. P. Bourguignon et H. Karcher construisent dans [2] un tenseur de courbure d'Einstein de dimension 6 à courbure sectioninelle positive dont l'intégrand d'Euler est négatif. Ils prennent une combinason linéaire des tenseurs de courbure des espaces symétriques irréductibles de dimension 6, soit

$$
R=\lambda R_{S^{6}}+\mu R_{\mathbf{C} P^{3}}+\nu R_{\mathbf{R} G^{2,5}},
$$

où $R_{S^{6}}$ désigne le tenseur de courbure de la sphère $S^{6}, R_{\mathbf{C} P^{3}}$ celui de l'espace projectif complexe $\mathbf{C} P^{3}$ et $R_{\mathbf{R} G^{2,5}}$ celui de la grassmannienne des 2-plans de $\mathbf{R}^{5}$. $\mathrm{Si}$ on remplace un des paramètres par la constante d'Einstein $\rho$ dans la formule donnant l'intégrand d'Euler, on obtient un polynôme du troisième degré en $\rho$ dont tous les coefficients ne sont pas identiquement nuls. Nous pouvons donc obtenir par exemple:

(i) des tenseurs de courbure d'Einstein de dimension 6 à intégrand d'Euler nul et constante d'Einstein non nulle (le polynôme en $\rho$ ne se réduit pas à son terme de plus haut degré);

(ii) un tenseur de courbure non nul de dimension 6 à courbure de Ricci nulle et à intégrand d'Euler nul (il suffit de faire $\rho=0$ dans le polynôme et d'ajuster les paramètres $\lambda$ et $\mu$ pour que le terme constant du polynôme soit nul; ceci est 
possible puisque ce terme est un polynôme homogène de degré 3 en $\lambda$ et $\mu$ ), d'où un contre-exemple à une généralisation du cas d'égalité de $\left(\mathrm{AE}_{4}^{\prime}\right)$.

Une autre façon beaucoup plus implicite d'utiliser l'approche (1) consiste à prendre des métriques données sur des variétés par des méthodes globales. Ainsi la solution de la conjecture de Calabi (cf. [16]) permet d'affirmer l'existence de métriques de Kähler-Einstein sur les hypersurfaces complexes $M$ de $\mathbf{C} P^{4}$ à première classe de Chern négatives ou nulle, i.e. celles dont le degré $d$ est supérieur ou égal à 5 .

Il est facile de calculer la caractéristique d'Euler d'une telle hypersurface $M^{d}$ puisqu'elle s'identifie à $c_{3}\left(M^{d}\right)$ : on a

$$
\chi\left(M^{d}\right)=d^{4}-5 d^{3}+10 d^{2}-10 d+5 .
$$

On voit facilement que $\chi\left(M^{d}\right) \geqslant \chi\left(M^{5}\right)=205$ pour $d \geqslant 5$.

Il existe donc un point $x$ de $M$ où l'intégrand d'Euler $\chi\left(R_{d}\right)$ est positif. En prenant le tenseur de courbure $-R_{d}(x), d \geqslant 5$, on a donc un exemple de tenseur de courbure d'Einstein à constante d'Einstein positive et à intégrand d'Euler négatif. De plus pour $d=5,-R_{5}$ est Ricci-plat et vérifie $\chi\left(-R_{5}\right)>0$.

Nous passons maintenant à la dimension 8 où nous produisons un contreexemple à $\left(\mathrm{AE}_{8}^{\prime}\right)$ en suivant la méthode (2).

Nous partons de la remarque suivante: si $z_{1}$ et $z_{2}$ sont deux tenseurs symétriques, alors la courbure de Ricci du tenseur de courbure $z_{1} \bullet z_{2}$ vaut $\operatorname{trace}\left(z_{1}\right) z_{2}+\operatorname{trace}\left(z_{2}\right) z_{1}-z_{1} \circ z_{2}-z_{2} \circ z_{1}$ où $\circ$ désigne la composition des endomorphismes.

Ainsi $z_{1} \bullet z_{2}$ est un tenseur d'Einstein dès qu'il vérifie les propriétés trace $\left(z_{1}\right)$ $=\operatorname{trace}\left(z_{2}\right)=0$ et $z_{1} \circ z_{2}=k$ Id où $k$ est une constante.

Dans une base orthonormée $\left(e_{i}\right)$ de $V$ nous définissons deux tenseurs symétriques $z_{1}^{\prime}$ et $z_{2}^{\prime}$ par

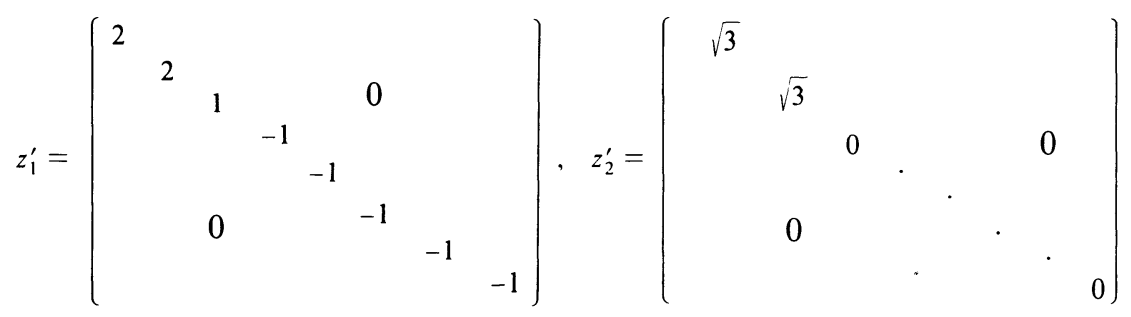

de telle sorte que $z_{1}=z_{1}^{\prime}+z_{2}^{\prime}$ et $z_{2}=z_{1}^{\prime}-z_{2}^{\prime}$ vérifient les conditions mentionnées ci-dessus. La constante d'Einstein de $R$ vaut 16/7 et les 2-vecteurs $e_{i} \wedge e_{j}$ forment une base de vecteurs propres de $R$ pour les valeurs propres 
$a_{12}=14, a_{13}=a_{23}=4, a_{1 \alpha}=a_{2 \alpha}=-4, a_{3 \alpha}=-2$ pour $4 \leqslant \alpha \leqslant 8$. On obtient ainsi

$$
\chi(R)=-2^{4} \cdot 3^{2} \cdot 5^{2}
$$

d'où le contre-exemple cherché.

Par ailleurs $R-\frac{2}{7} R_{S^{8}}$ est à courbure de Ricci nulle et pour ce tenseur nous avons

$$
\chi\left(R-\frac{2}{7} R_{S^{8}}\right)=-2^{10} \cdot 3 \cdot 5 \cdot 19 \cdot 7^{-3},
$$

d'où un exemple de tenseur de courbure de dimension 8 à courbure de Ricci nulle et intégrand d'Euler négatif.

\section{Contre-exemples kählériens en dimension 8}

Nous nous proposons de construire un tenseur de courbure de Kähler-Einstein de dimension complexe 4 pour lequel

$$
\kappa(R)>5^{4} \tau(R),
$$

où $\kappa(R)$ désigne le produit de la constante d'Einstein par $2 \pi$ et $\tau(R)$ l'intégrand du genre de Todd.

L'inégalité que nous voulons satisfaire s'exprime, en terme de classes de Chern, de la façon suivante:

$$
c_{1}^{4}>5^{3} \cdot 2^{-4} \cdot 3^{-2}\left(-c_{4}+c_{3} c_{1}+3 c_{2}^{2}+4 c_{2} c_{1}^{2}-c_{1}^{4}\right) .
$$

En utilisant les relations universelles entre classes de Pontrjaguine et classes de Chern, nous obtenons

$$
\begin{aligned}
& p_{1}^{2}=4 c_{2}^{2}-4 c_{2} c_{1}^{2}+c_{1}^{4}, \\
& p_{2}=2 c_{4}-2 c_{1} c_{3}+c_{2}^{2} .
\end{aligned}
$$

L'inégalité précédente peut donc se réécrire sous la forme

$$
3027 c_{1}^{4}>7500 c_{1}^{2} c_{2}+875 p_{1}^{2}-500 p_{2} .
$$

Il est raisonnable de partir du tenseur de courbure de l'espace projectif complexe $\mathbf{C} P^{4}$ que nous notons $R_{\mathbf{C} P^{4}}$, puisque pour cet espace il y a égalité, et que, $\mathbf{C} P^{4}$ étant un espace symétrique, les intégrands des nombres caractéristiques sont constants.

L'espace euclidien $(V,\langle\rangle$,$) est muni d'une structure complexe compatible$ avec la métrique et notée $J$. Nous désignons par j l'élément de $S^{2} \Lambda^{2} V$ défini pour $x$ et $y$ dans $V$ par

$$
j(x \wedge y)=J x \wedge J y
$$


Pour être kählérien le tenseur de courbure $R$ doit vérifier la relation $R \circ j=R$. Ceci s'exprime encore, de façon équivalente, en disant que $R$ opère dans le sous-espace vectoriel $\Lambda_{J}^{2} V$ de dimension $m^{2}$ dont les éléments sont les 2-formes $\omega$ vérifiant $\omega \circ J=J \circ \omega$.

Soit $\left(e_{1}, e_{2}, e_{3}, e_{4}, J e_{1}, J e_{2}, J e_{3}, J e_{4}\right)$ une base orthonormée adaptée de $V$. Les vecteurs suivants forment une base orthonormée de $\Lambda_{J}^{2} V$ de vecteurs propres de $R_{\mathbf{C} P^{4}}$ :

$$
\begin{aligned}
\sqrt{2} \omega_{1} & =e_{1} \wedge e_{2}+J e_{1} \wedge J e_{2}, \quad \sqrt{2} \tilde{\omega}_{1}=e_{3} \wedge e_{4}+J e_{3} \wedge J e_{4}, \\
\sqrt{2} \omega_{2} & =e_{1} \wedge e_{3}+J e_{1} \wedge J e_{3}, \quad \sqrt{2} \tilde{\omega}_{2}=e_{2} \wedge e_{4}+J e_{2} \wedge J e_{4}, \\
\sqrt{2} \omega_{3} & =e_{1} \wedge e_{4}+J e_{1} \wedge J e_{4}, \quad \sqrt{2} \tilde{\omega}_{3}=e_{2} \wedge e_{3}+J e_{2} \wedge J e_{3}, \\
\sqrt{2} \pi_{1} & =e_{1} \wedge J e_{2}+e_{2} \wedge J e_{1}, \quad \sqrt{2} \tilde{\pi}_{1}=e_{3} \wedge J e_{4}+e_{4} \wedge J e_{3}, \\
\sqrt{2} \pi_{2} & =e_{1} \wedge J e_{3}+e_{3} \wedge J e_{1}, \quad \sqrt{2} \tilde{\pi}_{2}=e_{2} \wedge J e_{4}+e_{4} \wedge J e_{2}, \\
\sqrt{2} \pi_{3} & =e_{1} \wedge J e_{4}+e_{4} \wedge J e_{1}, \quad \sqrt{2} \tilde{\pi}_{3}=e_{2} \wedge J e_{3}+e_{3} \wedge J e_{2}, \\
2 \phi_{0} & =e_{1} \wedge J e_{1}+e_{2} \wedge J e_{2}+e_{3} \wedge J e_{3}+e_{4} \wedge J e_{4}, \\
2 \tilde{\phi} & =e_{1} \wedge J e_{1}+e_{2} \wedge J e_{2}-e_{3} \wedge J e_{3}-e_{4} \wedge J e_{4}, \\
\sqrt{2} \phi_{1} & =e_{1} \wedge J e_{1}-e_{2} \wedge J e_{2}, \\
\sqrt{2} \tilde{\phi}_{1} & =e_{3} \wedge J e_{3}-e_{4} \wedge J e_{4} .
\end{aligned}
$$

On a, pour $R_{\mathbf{C} P^{4}}$,

$$
\begin{array}{llll}
R \omega_{i}=4 \omega_{i}, & R \tilde{\omega}_{i}=4 \tilde{\omega}_{i}, & R \pi_{i}=4 \pi_{i}, & R \tilde{\pi}_{i}=4 \tilde{\pi}_{i}, \\
R \phi_{0}=20 \phi_{0}, & R \tilde{\phi}_{0}=4 \tilde{\phi}_{0}, & R \phi_{1}=4 \phi_{1}, & R \tilde{\phi}_{1}=4 \tilde{\phi}_{1} .
\end{array}
$$

En fait, pour simplifier les calculs, on divisera toutes ces valeurs par 4.

On va chercher un tenseur de courbure $R$ admettant les vecteurs précédents comme vecteurs propres et ayant pour valeurs propres les nombres $\boldsymbol{\theta}, \tilde{\boldsymbol{\theta}}, p, \tilde{p}, f_{0}, f$, définis par

$$
\begin{array}{llll}
R \omega_{i}=\theta \omega_{i}, & R \tilde{\omega}_{i}=\tilde{\theta} \tilde{\omega}_{i}, & R \pi_{i}=p \pi_{i}, & R \tilde{\pi}_{i}=\tilde{p} \tilde{\pi}_{i}, \\
R \phi_{0}=f_{0} \phi_{0}, & R \tilde{\phi}_{0}=f \tilde{\phi}_{0}, & R \phi_{1}=f \phi_{1}, & R \tilde{\phi}_{1}=f \tilde{\phi}_{1} .
\end{array}
$$

On obtient $R_{\mathbf{C} P^{4}}$ en faisant $\theta=\tilde{\theta}=p=\tilde{p}=f=1, f_{0}=5$.

Pour ce tenseur de courbure on calculera successivement $p_{2}(R), p_{1}^{2}(R)$, $c_{1}^{2} c_{2}(R)$ et $c_{1}^{4}(R)$ en tenant compte des relations imposées par la première identité de Bianchi, relations qui s'écrivent

$$
\begin{aligned}
& \boldsymbol{\theta}=\frac{1}{2}\left(f_{0}-f-2 p\right), \\
& \tilde{\boldsymbol{\theta}}=\frac{1}{2}\left(f_{0}-f-2 \tilde{p}\right) .
\end{aligned}
$$


Nous commençons par évaluer $p_{2}(R)=\beta_{2,8} \operatorname{proj}_{\Lambda^{4} V}^{\perp}\left(R^{\cdot 2} \circ R^{\cdot 2}\right)$. Il nous faut donc calculer $R^{\cdot 2}$, puis $R^{\cdot 2} \circ R^{\cdot 2}$. Le coefficient $\beta_{2,8}$ est obtenu en normalisant sur $\mathbf{C} P^{4}$ pour lequel $p_{2}\left(\mathbf{C} P^{4}\right)=10$. Après un long calcul, nous obtenons

$$
\begin{aligned}
p_{2}(R)=2^{-5} & \left\{\frac{1}{4}\left(f-f_{0}\right)\left(7 f^{3}-f^{2} f_{0}-3 f_{0}^{2} f-3 f_{0}^{3}\right)+8 p \tilde{p} \theta \tilde{\theta}\right. \\
& +\frac{1}{2}\left(f-f_{0}^{2}\right)^{2}\left(p^{2}+\tilde{p}^{2}+\theta^{2}+\tilde{\theta}^{2}\right)+6\left(\theta^{2} \tilde{\theta}^{2}+p^{2} \tilde{p}^{2}\right. \\
& \left.+\tilde{\theta}^{2} p^{2}+\theta^{2} \tilde{p}^{2}\right)+\left(f-f_{0}\right)\left[\left(3 f+f_{0}\right)\left(p^{2}+\tilde{p}^{2}+\theta^{2}+\tilde{\theta}^{2}\right)\right. \\
& \left.\left.+2 p(3 \theta \tilde{\theta}+p \tilde{p})+\tilde{p}\left(3 \theta^{2}+3 \tilde{\theta}^{2}+p^{2}+\tilde{p}^{2}\right)\right]\right\} J^{4},
\end{aligned}
$$

où $J^{4}=J \wedge J \wedge J \wedge J$, et où nous avons effectué une nouvelle normalisation en prenant le volume de $\mathbf{C} P^{4}$ égal à 1 .

Pour évaluer $p_{1}(R)$, nous partons de la relation

$$
p_{1}(R)=\beta_{1,8} \operatorname{proj}_{\Lambda^{4} V}^{\perp}(R \circ R) .
$$

Nous obtenons

$$
\begin{aligned}
p_{1}(R)=\beta_{1,8}\left\{\theta^{2} \sum_{i=1}^{3} \omega_{i} \wedge \omega_{i}+\tilde{\theta}^{2} \sum_{i=1}^{3} \tilde{\omega}_{i} \wedge \tilde{\omega}_{i}+p^{2} \sum_{i=1}^{3} \pi_{i} \wedge \pi_{i}+\tilde{p}^{2} \sum_{i=1}^{3} \tilde{\pi}_{i} \wedge \tilde{\pi}_{i}\right. \\
\left.\quad+f_{0} \phi_{0} \wedge \phi_{0}+f\left(\tilde{\phi}_{0} \wedge \tilde{\phi}_{0}+\phi_{1} \wedge \phi_{1}+\tilde{\phi}_{1} \wedge \tilde{\phi}_{1}\right)\right\} \\
=\beta_{1,8}\left\{( \theta ^ { 2 } + p ^ { 2 } - \frac { 1 } { 2 } f _ { 0 } ^ { 2 } + \frac { 1 } { 2 } f ^ { 2 } ) \left(e_{1} \wedge e_{2} \wedge J e_{1} \wedge J e_{2}+e_{1} \wedge e_{3} \wedge J e_{1}\right.\right. \\
\left.\wedge J e_{3}+e_{1} \wedge e_{4} \wedge J e_{1} \wedge J e_{4}\right)+\left(\tilde{\theta}^{2}+\tilde{p}^{2}-\frac{1}{2} f_{0}^{2}+\frac{1}{2} f^{2}\right)\left(e_{2} \wedge e_{3}\right. \\
\left.\left.\wedge J e_{2} \wedge J e_{3}+e_{2} \wedge e_{4} \wedge J e_{2} \wedge J e_{4}+e_{3} \wedge e_{4} \wedge J e_{3} \wedge J e_{4}\right)\right\} .
\end{aligned}
$$

En normalisant sur $\mathbf{C} P^{4}$ nous obtenons

$$
p_{1}^{2}(R)=\frac{1}{4}\left(\theta^{2}+p^{2}-\frac{1}{2} f_{0}^{2}+\frac{1}{2} f^{2}\right)\left(\tilde{\theta}^{2}+\tilde{p}^{2}-\frac{1}{2} f_{0}^{2}+\frac{1}{2} f^{2}\right) J^{4} .
$$

Nous avons ensuite

$$
c_{2}=\frac{1}{2}\left(c_{1}^{2}-p_{1}\right) \text {. }
$$

Or, lorsque l'on prend le volume de $\mathbf{C} P^{4}$ égal à 1 , on a pour un tenseur de Kähler-Einstein $c_{1}(R)=f_{0} J$. D'où

$$
c_{1}^{2} c_{2}(R)=\frac{1}{8} f_{0}^{2}\left(\theta^{2}+\tilde{\theta}^{2}+p^{2}+\tilde{p}^{2}+f^{2}+3 f_{0}^{2}\right) J^{4} .
$$

Posons

$$
P(R)=7500 c_{1}^{2}(R)+875 p_{1}^{2}(R)-500 p_{2}(R)-3027 c_{1}^{4}(R) .
$$

Alors, en tenant compte des relations entre $p, \tilde{p}, \boldsymbol{\theta}, \tilde{\boldsymbol{\theta}}, f_{0}$ et $f$ exprimant la première identité de Bianchi, nous obtenons 


$$
\begin{aligned}
P(R)=5^{3} \cdot 2^{-2}\{8 & , 136 f^{4}+2 f^{4}-27 f_{0}^{3} f+41 f_{0}^{2} f^{2}-f_{0} f^{3}+12 p^{2} \tilde{p}^{2} \\
& +\left(f-f_{0}\right)\left(6 p \tilde{p}^{2}-2 \tilde{p}^{3}\right)+2\left(p^{2}+\tilde{p}^{2}\right)\left(2 f^{2}+27 f_{0}^{2}+f f_{0}\right) \\
& \left.+(p+\tilde{p})\left(-27 f_{0}^{3}+26 f_{0}^{2} f-f_{0} f^{2}+2 f^{3}\right)\right\} .
\end{aligned}
$$

Nous pouvons trouver différents quadruplets $\left(p, \tilde{p}, f_{0}, f\right)$ rendant $P$ négatif. C'est le cas de $(2,-27,-1,-7)$ et $\left(-\frac{1}{2}, 39 / 2,0,-1\right)$. Ce polynôme est aussi négatif dans les régions

$$
\begin{aligned}
& p=0, \quad \tilde{p}=1, \quad-0,36365 \leqslant f_{0}<0, \quad f=0, \\
& p=0, \quad \tilde{p}=1, \quad f_{0}=0, \quad 0<f \leqslant 0,3926 .
\end{aligned}
$$

Nous avons donc trouvé les exemples cherchés. On peut vérifier que pour le polynôme $P$ le point $(1,1,5,1)$ correspondant à $R_{\mathrm{C} P^{4}}$ est un minimum relatif. Il serait intéressant d'établir cette propriété de minimum relatif pour tout l'espace des tenseurs de courbure de Kähler-Einstein car cela fournirait une autre preuve de l'isolation de la métrique de Fubini-Study parmi les métriques de Kähler-Einstein sur $\mathbf{C} P^{4}$.

On peut encore utiliser l'exemple précédent pour exhiber un tenseur de courbure de Kähler-Einstein non nul à courbure de Ricci nulle et intégrand d'Euler nul.

Pour avoir $c_{1}(R)=0$, il faut prendre $f_{0}=0$. Nous avons par ailleurs

$$
\chi=c_{4}=\frac{1}{2}\left(p_{2}-\frac{1}{4} p_{1}^{2}\right)
$$

d'où

$$
\begin{aligned}
\chi(R)=2^{-6} & {\left[\frac{7}{4} f^{4}+8 p \tilde{p} \theta \tilde{\theta}+\frac{1}{2} f^{2}\left(p^{2}+\tilde{p}^{2}+\theta^{2}+\tilde{\theta}^{2}\right)+6\left(\theta^{2} \tilde{\theta}^{2}+p^{2} \tilde{p}^{2}\right.\right.} \\
& \left.+\tilde{\theta}^{2} p^{2}+\theta^{2} \tilde{p}^{2}\right)+f\left\{3 f\left(p^{2}+\tilde{p}^{2}+\theta^{2}+\tilde{\theta}^{2}\right)\right. \\
& \left.\left.+2 p(3 \theta \tilde{\theta}+p \tilde{p})+\tilde{p}\left(3 \theta^{2}+3 \tilde{\theta}^{2}+p^{2}+\tilde{p}^{2}\right)\right\}\right] \\
& -2^{-5}\left(\theta^{2}+p^{2}+\frac{1}{2} f^{2}\right)\left(\tilde{\theta}^{2}+\tilde{p}^{2}+\frac{1}{2} f^{2}\right)
\end{aligned}
$$

On remarque que si $\tilde{\theta}=-\tilde{p}, \theta=p=f=0$, alors $R$ est un tenseur de courbure kählérien non nécessairement nul et dont l'intégrand d'Euler est nul.

Bien sûr, par perturbation, en prenant par exemple $f=2 \varepsilon, p=\varepsilon, \theta=0$, $\tilde{\theta}=1-\varepsilon, p=1$, on a $\chi(R) \sim 2^{-3} \varepsilon$ lorsque $\varepsilon$ tend vers 0 , d'où des exemples avec $\chi(R)<0$.

\section{Références}

[1] M. Berger, Sur les variétés d'Einstein compactes, C. R. III ${ }^{e}$ Réunion Math. Expression Latine, Louvain Belgique, 1966, 35-55.

[2] J. P. Bourguignon \& H. Karcher, Curvature Operators: Pinching estimates and geometric examples, Ann. Sci. École Norm. Sup. 11 (1978) 71-92. 
[3] S. S. Chern, On the curvature and characteristic ciasses of a Riemannian manifold, Abh. Math. Sem. Univ. Hamburg 20 (1956) 117-126.

[4] R. Geroch, Positive sectional curvature does not imply positive Gauss-Bonnet integrand, Proc. Amer. Math. Soc. 54 (1976) 267-270.

[5] N. Hitchin, Compact four-dimensional Einstein manifolds, J. Differential Geometry 9 (1974) 435-441.

[6] D. L. Johnson, Curvature and Euler characteristic for six-dimensional Kähler manifolds, preprint.

[7] P. Klembeck, On Geroch's counterexample to the algebraic Hopf conjecture, Proc. Amer. Math. Soc. 59 (1976) 334-336.

[8] R. S. Kulkarni, On the Bianchi identities, Math. Ann. 199 (1972) 175-204.

[9] S. Mori, Projective manifolds with ample tangent bundles, Ann. of Math. 110 (1979) 590-606.

[10] A. Polombo, Nombres caractéristiques d' une variété riemannienne de dimension 4, J. Differential Geometry 13 (1978) 145-162.

[11] I. M. Singer \& J. A. Thorpe, The curvature of 4-dimensional Einstein spaces, Global Analysis, Papers in Honor of K. Kodaira, Princeton University Press, Princeton, 1969, 355-365.

[12] Y. T. Siu \& S. T. Yau, Compact Kähler manifolds of positive bisectional curvature, Invent. Math. 59 (1980) 189-204.

[13] A. Stehney, Courbure d'ordre $p$ et les classes de Pontrjagin, J. Differential Geometry 8 (1973) 125-133.

[14] J. A. Thorpe, Some remarks on the Gauss-Bonnet integral, J. Math. Mech. 18 (1969) 779-786.

[15] J. Vilms, On curvature operators of bounded rank, Preprint, Colorado State University, Fort Collins.

[16] S. T. Yau, On Calabi's conjecture and some new results in algebraic geometry, Proc. Nat. Acad. Sci. U.S.A. 74 (1977) 1798-1799. 